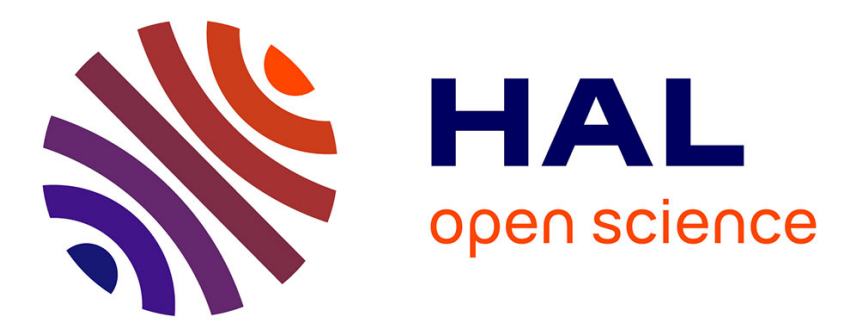

\title{
DISPOSABLE SYSTEM FOR IN-VIVO MECHANICAL CHARACTERIZATION OF SOFT TISSUES BASED ON VOLUME MEASUREMENT
}

Seyed Ali Elahi, Nathanaël Connesson, Yohan Payan

\section{- To cite this version:}

Seyed Ali Elahi, Nathanaël Connesson, Yohan Payan. DISPOSABLE SYSTEM FOR INVIVO MECHANICAL CHARACTERIZATION OF SOFT TISSUES BASED ON VOLUME MEASUREMENT. Journal of Mechanics in Medicine and Biology, 2018, 18 (04), pp.1850037. 10.1142/S0219519418500379 . hal-01930070

\section{HAL Id: hal-01930070 https://hal.science/hal-01930070}

Submitted on 21 Nov 2018

HAL is a multi-disciplinary open access archive for the deposit and dissemination of scientific research documents, whether they are published or not. The documents may come from teaching and research institutions in France or abroad, or from public or private research centers.
L'archive ouverte pluridisciplinaire HAL, est destinée au dépôt et à la diffusion de documents scientifiques de niveau recherche, publiés ou non, émanant des établissements d'enseignement et de recherche français ou étrangers, des laboratoires publics ou privés. 
Journal of Mechanics in Medicine and Biology

DOI: $10.1142 / \mathrm{S} 0219519418500379$

\title{
Disposable system for in-vivo mechanical characterization of soft tissues based on volume measurement
}

\author{
SEYED ALI ELAHI \\ TIMC-IMAG Laboratory, UMR CNRS 5525, Grenoble Alpes University, Pavillon Taillefer, \\ Faculty of Medicine, Domaine de la Merci \\ La Tronche cedex, 38706, France \\ seyed-ali.elahi@univ-grenoble-alpes.fr \\ NATHANAEL CONNESSON \\ TIMC-IMAG Laboratory, UMR CNRS 5525, Grenoble Alpes University, Pavillon Taillefer, \\ Faculty of Medicine, Domaine de la Merci \\ La Tronche cedex, 38706, France \\ nathanael.connesson@gmail.com \\ YOHAN PAYAN \\ TIMC-IMAG Laboratory, UMR CNRS 5525, Grenoble Alpes University, Pavillon Taillefer, \\ Faculty of Medicine, Domaine de la Merci \\ La Tronche cedex, 38706, France \\ yohan.payan@univ-grenoble-alpes.fr \\ Received (Day Month Year) \\ Accepted (Day Month Year)
}

In-vivo characterization of soft tissues is a key step toward biomechanical simulation and planning of intra-operative assisted surgery. To achieve this, aspiration method is a standard technique: tissue is aspirated through a hole while measuring the pressure and associated apex height. An inverse problem is then solved to identify the material mechanical properties.

In the literature, the apex height is usually measured using a camera, which induces design difficulties, in particular in regards on the required sterilization process for in-vivo measurements.

In this paper, the idea is to replace the apex height optical measurement by the measurement of the aspirated tissue volume. The proposed method enables to reduce the system head to a simple tube: sterilizations becomes easy and the system is disposable after use. The proposed system is thus the simplest, lightest and cheapest one could achieve. It is also to the authors knowledge the first time ever in aspiration method that the aspired volume is the extracted data.

As the data signal-to-noise ratio is the main factor impacting any applied inverse method when extracting the mechanical properties, the aim of this work is to assess and compare the experimental signal-to-noise ratio in the raw volume measurements obtained either optically or with the method proposed. Explicit results of inverse methods using volumes as input data are not presented in this paper for concision purpose.

The effects on accuracy of various experimental parameters has been investigated and quantified: the volume measurement has proved to present a same order or even 
better signal-to-noise ratio compared to optical measurements.

Keywords: Suction/Aspiration Method; Soft Tissues Characterization; Experimental Mechanics; In-Vivo Measurement; Inverse Characterization.

10.400: Mechanics of the Soft Tissues

\section{Introduction}

Understanding and measuring human organs mechanical properties impacts medical practices ${ }^{1}$. In particular, such mechanical properties provide insights for clinical diagnosis ${ }^{2,3}$, surgical training (simulators) ${ }^{4}$, tissue replacement engineering ${ }^{5}$, and trauma research ${ }^{6}$. Since mechanical behavior of living tissues varies between invivo and ex-vivo conditions ${ }^{7}$, measuring these properties in-vivo and in-situ is important.

In order to perform intra-operative measurements on humans, the procedure must be non-traumatic and operated under sterile conditions. The proposed procedure must also comply with space and time limitations in the operating room. These constraints motivated researchers to design devices with small sizes, low weights and avoiding if possible any electronic parts, so as to be able to withstand severe sterilization processes.

To in-vivo and in-situ measure the sought mechanical properties, various methods have been proposed in the literature such as indentation ${ }^{8,9,10}$, grasping ${ }^{11}$, torsion ${ }^{12}$, and aspiration ${ }^{13,14}$. Among these methods, aspiration/suction is the most widely used technique due to its simplicity and robustness. Several commercial devices are designed for dermatology market (Cutometer from Courage and Khazaka, Germany, or Dermaflex from Cortex Technology, Denmark). In particular, aspiration method limits relative displacement of the tissues in relation with the device and offers a good control of the applied boundary conditions.

The principle of the aspiration method consists in putting a chamber with a hole in contact with the tissue and in setting a negative pressure inside the chamber. The tissue is thus aspirated inside the chamber through the hole while aspiration pressure is measured during the test (Fig. 1). Authors usually propose to measure the aspired height using different methods such as ultrasound ${ }^{13,15}$, mechanical stops ${ }^{16}$ or cameras, associated to mirrors or prisms $17,18,19,20,21,22,23,24,25$. Yet, as ultrasound methods are expensive and add additional experimental constraints, optical methods are usually preferred in practical cases to provide pressure versus apex height characteristic curves.

In optical methods, the process is observed by a camera, either directly (Fig. 1) or using a mirror or prism (Fig. 2a) : apex height of the aspired tissue inside the chamber is measured using recorded images. Diagram of pressure versus apex height of the aspired tissue can thus be plotted. Material properties of the tissue are then identified with these experimental data using inverse methods such as updated FE method ${ }^{18}$ or gap method ${ }^{26}$. For example, such aspiration devices have been used 
to estimate the mechanical properties of cervix ${ }^{16}$, facial tissues ${ }^{18,25}$, brain ${ }^{21}$ or liver 24,7 .

These examples of optical aspiration methods yet underline limitations:

(1) As a camera can not go through severe sterilization processes, its contact with tissues must be prevented, which constrained authors to propose complex designs, usually using mirrors or prisms $18,25,17,19,20,21,22,23,24$.

(2) The use of cameras and mirrors requires accurate relative positioning, which lead to design systems that are large and rigid ${ }^{17,22}$. These constraints prevents wide and easy use of these systems during surgery.

A typical example of design evolution is presented by Schiavone et al. ${ }^{20}$, where a light pipette and an external camera have been first used. This system was able to meet the required severe sterilization and handling issues imposed during surgery. Yet, using an external camera induced measurements errors due to misalignment. The authors thus designed a two part Light Aspiration device for in-vivo Soft TIssue Characterization (LASTIC) ${ }^{23,22}$ (Fig. 2a). The obtained device head was a $3 \mathrm{~cm} \times 3 \mathrm{~cm}$ metallic cylinder divided in two compartments with circular aspiration aperture (diameter of $12 \mathrm{~mm}$ ). The sterilizable lower compartment was a cylindrical chamber made airtight by a glass window in which a negative pressure was applied. A miniature digital camera was fixed inside an upper chamber (not sterilizable) and placed relative to the mirror so as to avoid misalignment errors.

This final LASTIC head has yet not been used in intra-operative situations due to its still relative complexity, large weight and large size compared to its aspiration aperture.

\section{Aspiration}

\section{Pressure}

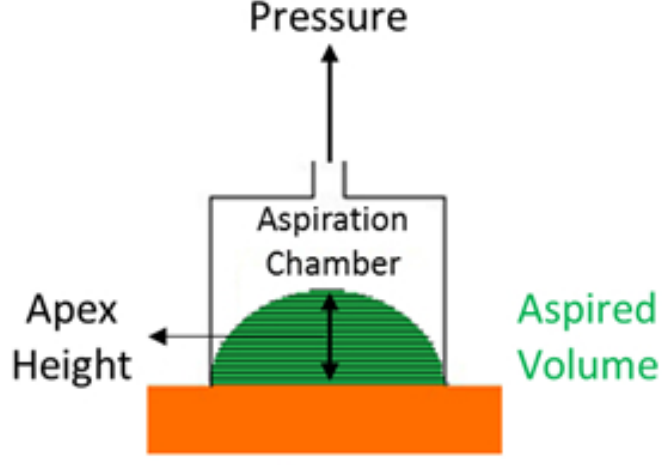

Tissue

Fig. 1. General scheme of the aspiration method. 
In this paper, the idea is to replace the apex height optical measurement by the measurement of the aspirated tissue volume. Such a change in the method enables to remove camera, mirror and all electronics from the system head that is basically reduced to a simple aperture and is thus able to meet the required severe sterilizations (Fig 2b). The proposed system is thus the simplest, lightest and cheapest one could achieve. According to the authors, thus simplifying the system will enable a generalized use of the device.

Switching the input data from apex height to volume measurement will only slightly change the inverse method process: both apex height and aspirated tissue volume are directly related to the aspirated tissue surface shape. The mechanical properties thus identified with either input data rely on the same hypotheses (boundary conditions, material thickness and anisotropy, mechanical properties gradients due to different layers, etc.) and will provide similar results. As these inverse method results have already been published and validated in the literature $17,16,18,25,24,21,7$, such inverse identification issues are considered as beyond the scope of this paper.

Eventually, only differences in the input data signal-to-noise ratio can significantly impact the inverse analyzes. The main question to deal with is then to assess if the experimental volume measurements signal-to-noise ratio obtained optically or with the proposed method are comparable: is there a loss of measurement precision while changing of measurement mean? This question will be answered experimentally by comparing volume measurements obtained either optically or with the proposed method.

The measurement method based on aspirated volume is first thoroughly presented (section 2). This method is then compared with a specifically designed reference method and then with more classical optical measurements (sections 3 and 4). The results obtained with each setup are then analyzed with a particular attention to reproducibility, error, measurement standard deviation (section 5), and impact of
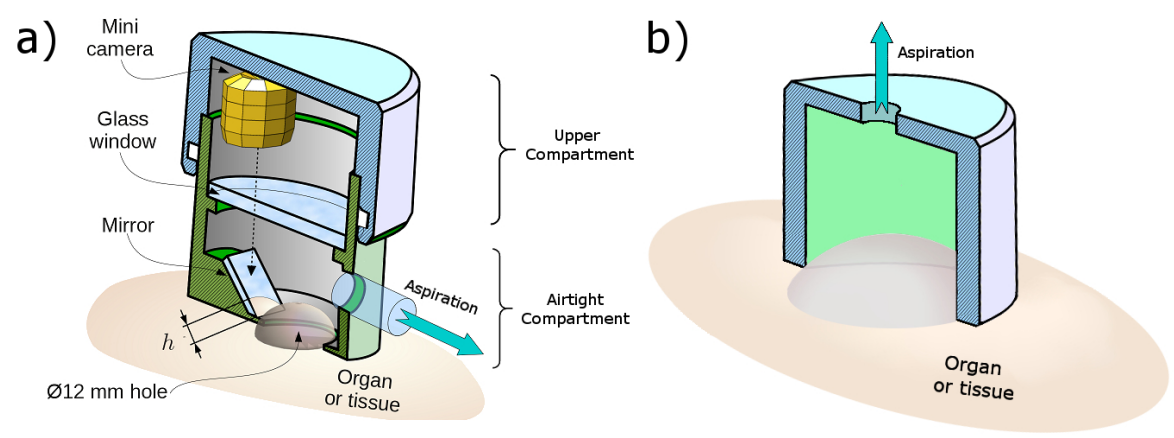

Fig. 2. a) Design of LASTIC head and its components ${ }^{23}$, b) Proposed simplified design. 
different setup experimental parameters (section 6). Finally, some recommendations are provided to help users to obtain the same results quality (section 7 ).

\section{Rate-based volume measurement method}

During in-situ and in-vivo tests, the proposed new system is simply composed of a syringe pump, a manometer and an aspiration chamber (Fig. 3).

The volume removed by the syringe pump $V^{\text {total }}$ and the associated pressure $P$ are measured during the test. Yet, the volume removed by the syringe pump $V^{\text {total }}$ is obviously different from the tissue volume $V_{\text {sample }}$ : this difference is mainly due to volume changes in the system because of the applied pressure; the system volume $V_{\text {system }}$ is compressible due to air expansion and elasticity of the components (connections, tubes, syringe, etc.). The total removed volume $V^{\text {total }}$ can therefore be seen as the sum of the aspired tissue volume $V_{\text {sample }}^{R B}$ and the volume changes due to system compressibility $V_{\text {system }}$ :

$$
V^{\text {total }}(P)=V_{\text {sample }}^{R B}(P)+V_{\text {system }}(P)
$$

The system volume function $V_{\text {system }}(P)$ is experimentally assessed during a second step by testing an undeformable material, referred to as rigid test: in this experimental configuration the aspired tissue volume $V_{\text {sample }}^{R B}$ remains zero and the system volume compressibility $V_{\text {system }}$ can be directly measured using Eq. 1:

$$
V_{\text {system }}(P)=V_{\text {rigid }}^{\text {total }}(P)
$$

Combining the system volume $V_{\text {system }}$ (Eq. 2) and Eq. 1, the aspired tissue volume $V_{\text {sample }}^{R B}$ can thus naturally be estimated by:

$$
V_{\text {sample }}^{R B}(P)=V^{\text {total }}(P)-V_{\text {system }}(P)
$$

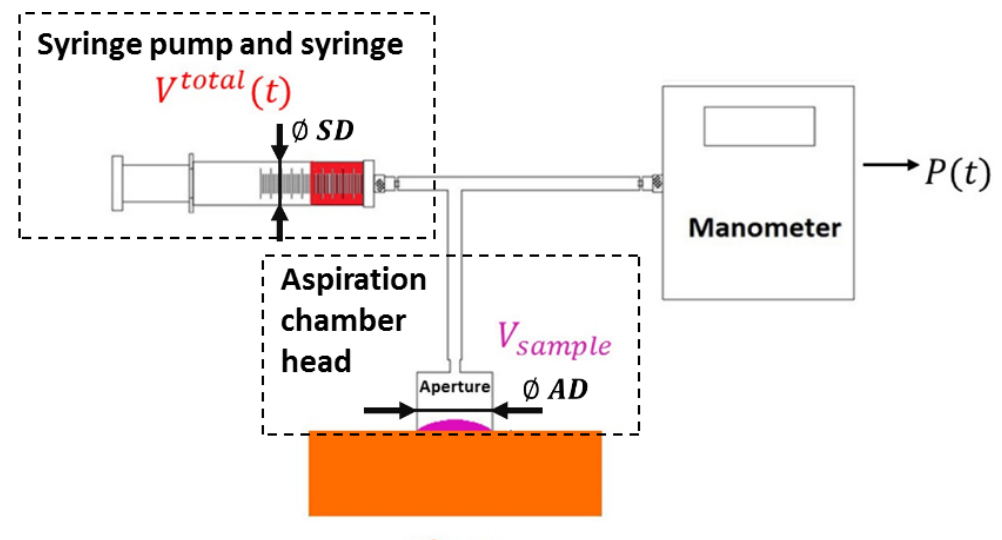

Tissue

Fig. 3. Measurement principle of the proposed rate-based volume measurement method. 
When applying the rate-based volume measurement method $V_{\text {sample }}^{R B}$ during invivo experiments, the total volume $V^{\text {total }}$ will be measured first, using a sterilized aspiration head. The rigid test will be performed afterward, when sterilized conditions are no longer required. During this test, a clamp system can be used to avoid leakage between the aspiration head and the undeformable material. Performing this additional rigid test adds less than 5 minutes to the overall procedure.

\section{Performance assessment test-bench}

As the aim of this work is to compare optical and rate-based volume measurement signal-to-noise ratio, all results should be compared with a reference obtained in identical conditions. Three different methods and associated volume measurements will thus be performed on the same material:

(1) the proposed rate-based volume measurement method $V_{\text {sample }}^{R B}$ (section 2),

(2) a reference method $V_{\text {sample }}^{\text {reference }}$ (section 3.2),

(3) and a camera based method $V_{\text {sample }}^{\text {camera }}($ section 3.3).

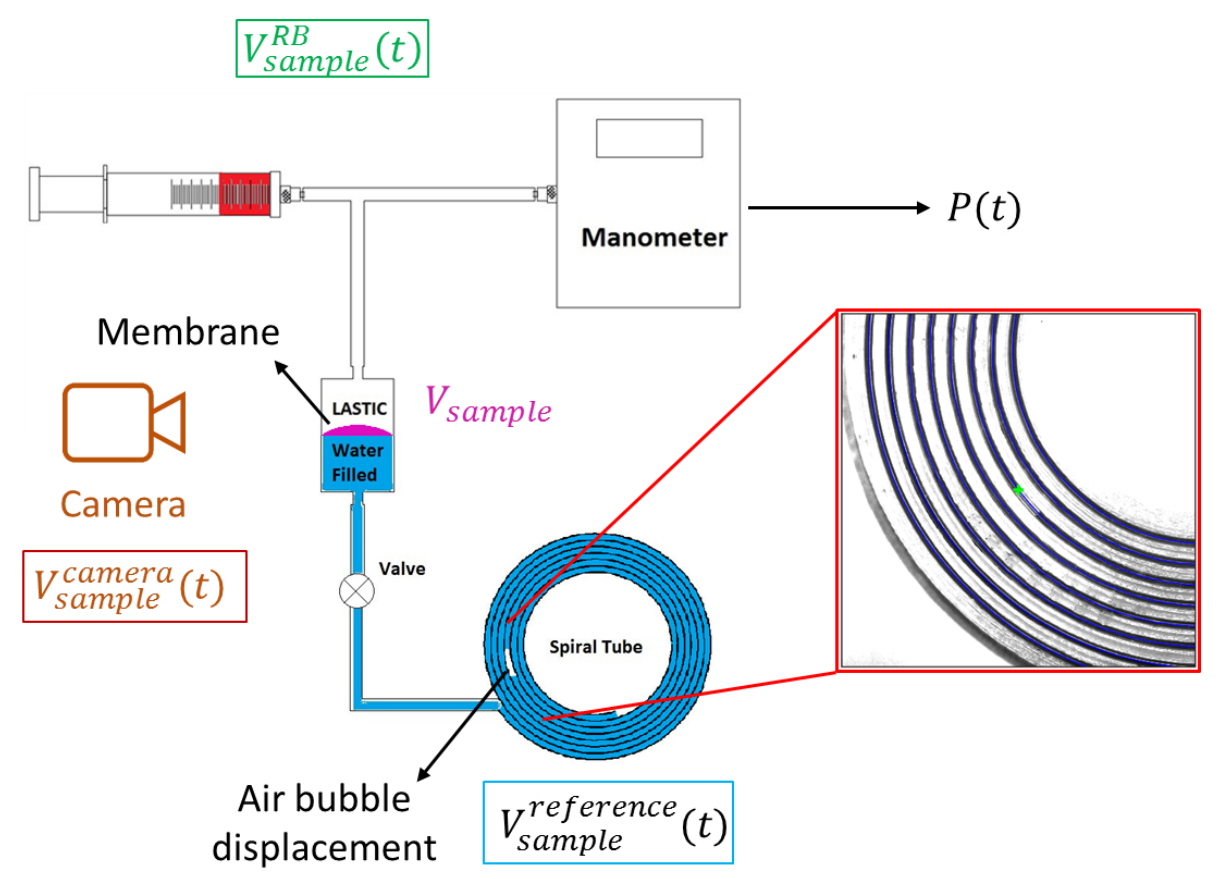

Fig. 4. Performance assessment configuration: simultaneous measurement of the aspirated tissue volume using the 3 methods: the reference method $V_{\text {sample }}^{\text {reference }}$, the rate-based method $V_{\text {sample }}^{R B}$ and the camera method $V_{\text {sample }}^{\text {camera }}$. 
In this work, it has been chosen to perform these three measurements simultaneously on the same tested material. As no inverse characterization will be performed here, the tested material is not required to be known or to mimic accurately the tissue mechanical behavior, but only to provide a sufficient shape variation (flat to a half sphere in this case) for a pressure range of the order of magnitude observed during actual tissue mechanical characterization: the tests have been performed in a range of 0 to 50 mbars, which is approximately the range obtained while characterizing in-vivo lower lip tissues ${ }^{18}$.

In the performance assessment configuration, the tissue has thus been replaced with a soft thin membrane cut from a latex glove (Fig. 4). This membrane has been glued to a system providing the reference volume $V_{\text {sample }}^{\text {reference }}$ (section 3.2 ). A camera has been used to take pictures of the aspired membrane during the tests and to deduce the aspired volume $V_{\text {sample }}^{\text {camera }}$ (section 3.3).

The provided results will thus provide the signal-to-noise ratio of the rate-based volume measurement method $V_{\text {sample }}^{R B}$ and classic optical method $V_{\text {sample }}^{\text {camera }}$ by using the reference volume $V_{\text {sample }}^{\text {reference }}$.

\subsection{Rate-based volume measurement method}

The method previously presented is directly applied (section 2). In particular, the rigid test in this test configuration is performed by closing the valve in the setup (Fig. 4), so that the contribution of the aspirated tissue volume is zero during the rigid test.

\subsection{Reference volume measurement}

A reference volume measurement device has been developed to estimate measurement errors (Fig. 4). Such a device obviously will not be used in practical cases.

This device, glued to a membrane, simply consists in a thin tube filled with water where an air bubble has been inserted (Fig. 5). Any movement of the membrane will induce movements of both the water and the bubble. Knowing the tube section $S$ and the bubble displacement $\Delta L$ straightforwardly provides the "tissue" aspirated volume $V_{\text {sample }}^{\text {reference }}$ :

$$
V_{\text {sample }}^{\text {reference }}=S \Delta L
$$

The bubble movements $\Delta L$ have been tracked on pictures synchronized with the pressure measurement. A dark background and proper lighting have been used to help automated bubble position tracking.

\subsection{Camera volume measurement method}

The proposed rate-based method $V_{\text {sample }}^{R B}$ should be compared to an optical based method $V_{\text {sample }}^{\text {camera }}$ even if the measurements using camera will not be necessary to 

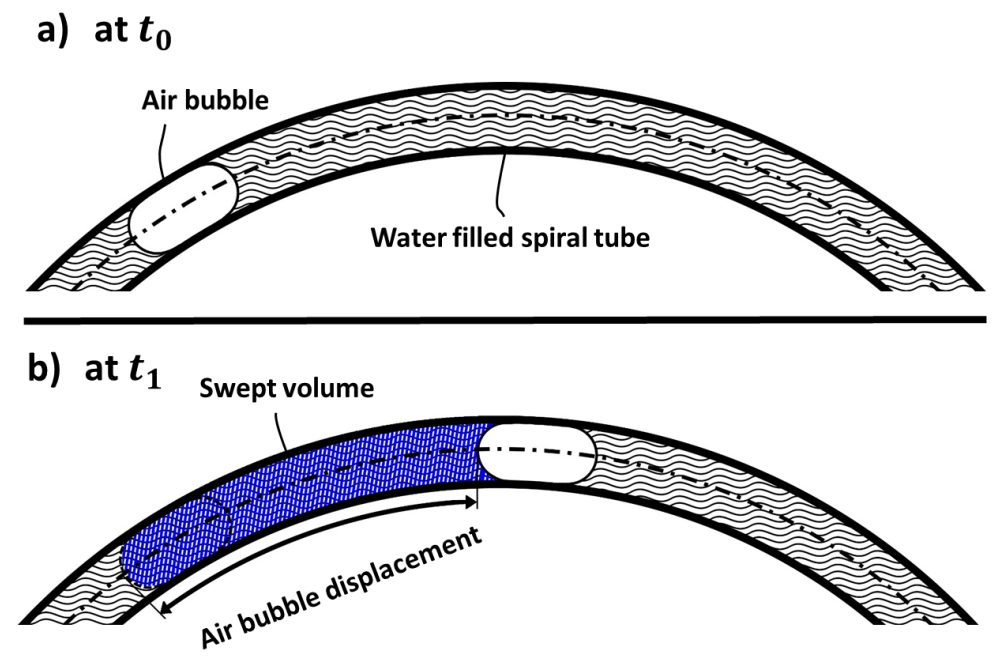

Fig. 5. Air bubble inside the spiral tube a) before and b) after the aspiration process.

apply the proposed method. For this, a camera has been used to take pictures of the aspired membrane shape $h(r)$ during the test (Fig. 6). Computing the associated volume can be easily performed by:

$$
V_{\text {sample }}^{\text {camera }}=\pi \int_{-R}^{R} r h(r) d r
$$

where $r$ is the radius of any point $\mathrm{M}$ of the aspirated surface, $d r$ is an elementary radius, $h$ is the membrane height above point $\mathrm{M}$, and $R$ is the aperture internal radius (Fig. 6c).

A dark background and proper lighting have been used to help automated membrane shape $h(r)$ recognition. According to Fig. 6b, some irregularities are visible in the lower part of the initial picture, which are due to the glue between the membrane and the aspiration chamber. The erroneous additional volume induced by these extra white parts is simply removed by subtracting the estimated volume of the initial picture from all other volumes during the aspiration.

\section{Preliminary results and parameters definition}

\subsection{Preliminary results}

An example of typical results obtained simultaneously by the three methods is presented as pressure $P$ versus volume curves (Fig. 7).

Curves 1 to 3 are obtained using the rate-based volume measurement 

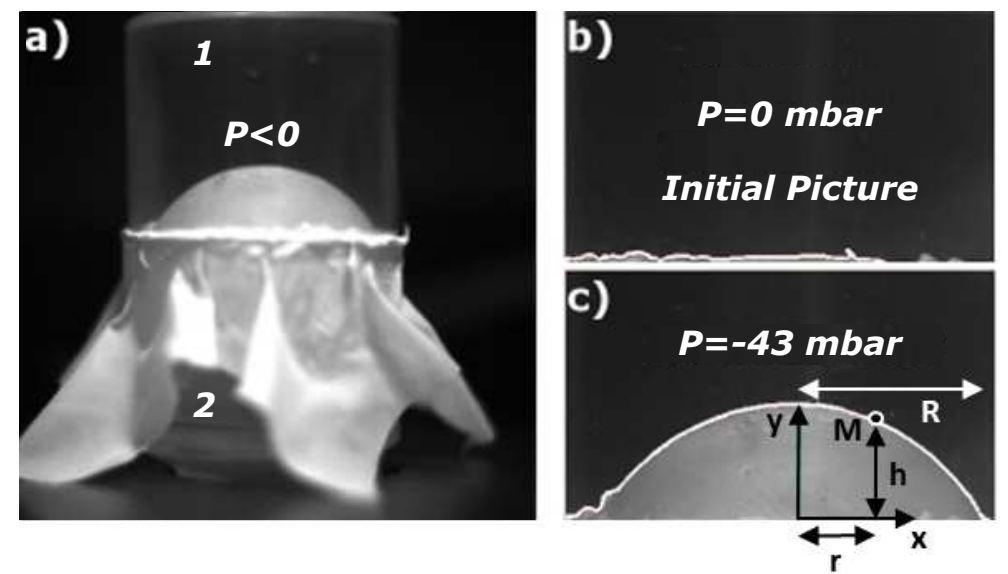

Fig. 6. a) Global view of the aspiration chamber: (1) aspiration head and (2) reference measurement system and pictures used to measure the aspired volume $V_{\text {sample }}^{\text {camera }}$ b) before and c) during aspiration.

method $V_{\text {sample }}^{R B}$ with a rate of $0.4 \mathrm{ml} / \mathrm{min}$, which proved to be small enough to provide quasi-static mechanical behavior:

- the total volume $V^{\text {total }}(P)$ obtained with the syringe pump while aspirating the membrane is presented as Curve 2 (Eq. 1)

- the measurement obtained during rigid test $V_{\text {rigid }}^{\text {total }}(P)$, valve closed, is presented as Curve 1 (Eq. 2). This volume variation is mainly due to the volume variation of air but also to the polyurethane tubes connecting the aspiration head, syringe pump and manometer. Special attention has been made to prevent any possible leakage in the connection zones.

- the sought volume $V_{\text {sample }}^{R B}$ (Curve 3) is the result of the difference of the two previous curves (Eq. 3).

Curves 4 and 5 are obtained using the reference $V_{\text {sample }}^{\text {reference }}$ and camera based methods $V_{\text {sample }}^{\text {camera }}$, respectively (Eq. 4 and 5, respectively). It should be specified here that the reference measurement system has been built using a tube of internal diameter of $0.9 \mathrm{~mm}$ to increase the bubble movement sensitivity. The bubble position has been estimated with a precision of about $\pm 1 \mathrm{~mm}$; the reference volume $V_{\text {sample }}^{\text {refere }}$ variations are thus measured with an accuracy of about $\pm 0.64 \mu l$.

These preliminary tests underline that all three pressure $P$ versus volume curves $\left(V_{\text {sample }}^{R B}, V_{\text {sample }}^{\text {reference }}, V_{\text {sample }}^{\text {camera }}\right)$ are very similar, validating thus the three proposed methods. The signal-to-noise ratio of each method needs now to be estimated, which will be presented in section 6 . 


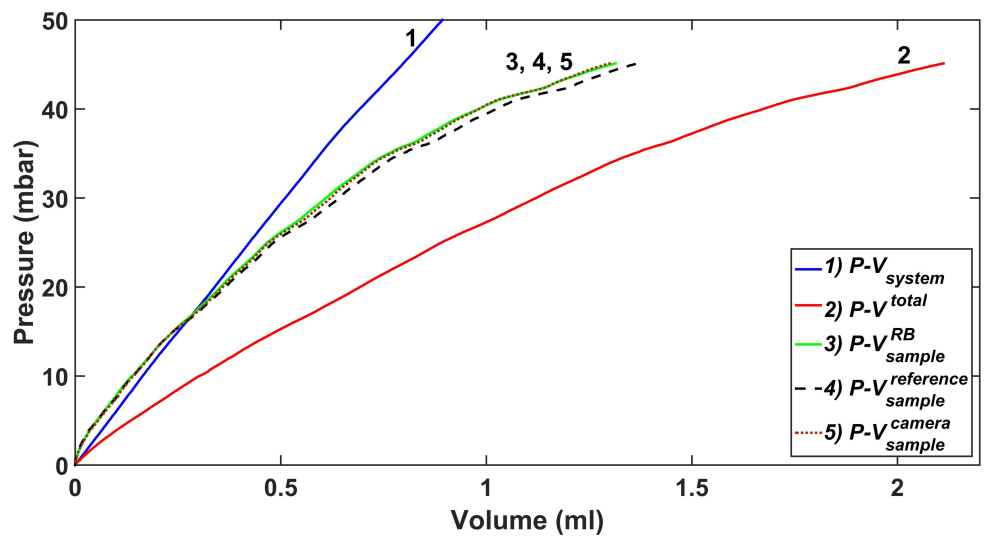

Fig. 7. Pressure versus volume for each experiment.

\subsection{Parameter definition and experimental design}

The signal-to-noise ratio of the rate-based volume method depends on the inner aspiration Aperture Diameter (AD) and the Syringe Diameter (SD) associated to the syringe pump (Fig. 3). Tests have thus been performed for two different inner Aperture Diameters (AD) and three different Syringe Diameters (SD). Therefore, 6 groups of tests have been conducted. Syringe and aperture inner diameters for the 6 test groups have been summarized in table 1. As the camera volume mea-

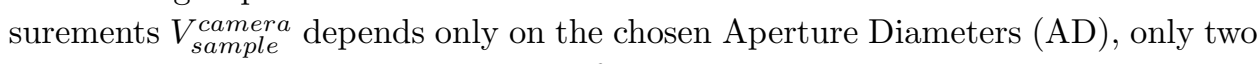
measurement sets are required to perform the signal-to-noise ratio study on the camera volume measurements.

\begin{tabular}{llllll}
\hline Test & AD & SD & \multicolumn{3}{c}{ Performed volume measurement method } \\
\cline { 4 - 6 } groups & $(\mathrm{mm})$ & $(\mathrm{mm})$ & $V_{\text {sample }}^{\text {reference }}$ & $V_{\text {sample }}^{R B}$ & $V_{\text {sample }}^{\text {camera }}$ \\
\hline VT-1 & 21.0 & 21.0 & $\checkmark$ & $\checkmark$ & $\checkmark$ \\
VT-2 & 21.0 & 12.9 & $\checkmark$ & $\checkmark$ & no data \\
VT-3 & 21.0 & 4.6 & $\checkmark$ & $\checkmark$ & no data \\
VT-4 & 12.9 & 21.0 & $\checkmark$ & $\checkmark$ & $\checkmark$ \\
VT-5 & 12.9 & 12.9 & $\checkmark$ & $\checkmark$ & no data \\
VT-6 & 12.9 & 4.6 & $\checkmark$ & $\checkmark$ & no data \\
\hline
\end{tabular}




\section{Error measurement tests}

In order to estimate the error of the method, the 6 test groups (table 1) have been repeated 9 times. Diagrams of pressure $P$ versus measured aspired volume using rate-based $V_{\text {sample }}^{R B}$, reference $V_{\text {sample }}^{\text {reference }}$ and camera $V_{\text {sample }}^{\text {camera }}$ methods for the 9 repetitions of test group VT-1 are presented in Fig. 8 as an example of raw data. The results show mechanical behavior differences for these successive tests, but, for each particular test, the three methods provide very similar results. This underlines that the rate-based method is able to monitor the lack of the chosen latex membrane mechanical reproducibility (attributed here to the complex local loading history and latex non linear behavior): the measurement error is lower than the mechanical behavior variations between tests.

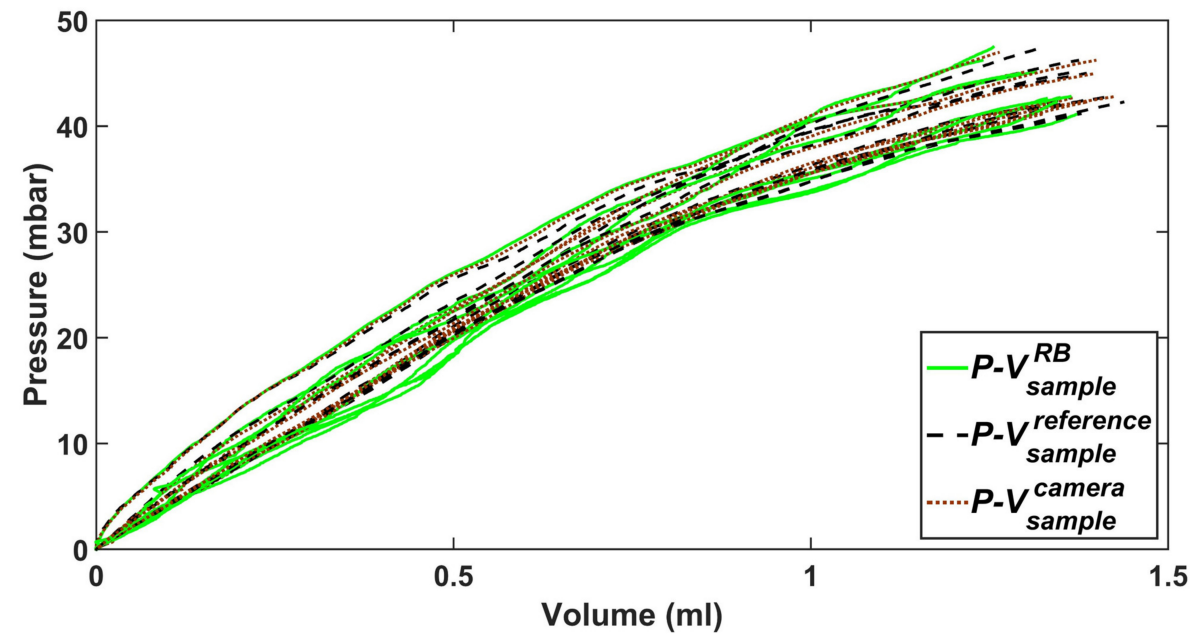

Fig. 8. Comparison of 9 diagrams of pressure $P$ versus measured aspired volume using rate-

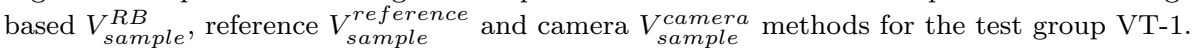

The errors of the rate-based volume measurement $V_{\text {sample }}^{R B}$ and of the camera $V_{\text {sample }}^{\text {camera }}$ methods have been computed using the reference volume $V_{\text {sample }}^{\text {refere }}$. Such errors are thus defined as:

$$
\begin{gathered}
\operatorname{Error}^{R B}(P)=V_{\text {sample }}^{R B}(P)-V_{\text {sample }}^{\text {reference }}(P) \\
\operatorname{Error}^{\text {camera }}(P)=V_{\text {sample }}^{\text {camera }}(P)-V_{\text {sample }}^{\text {reference }}(P)
\end{gathered}
$$

For example, the experimental results of Eq. 6 (Error $\left.{ }^{R B}\right)$ for test group VT-1 are presented versus pressure $P$ in Fig. 9. In addition, the associated STandard Deviations (STD) and average of volume measurements errors $\operatorname{Error}^{R B}$ have been 
reported as three thick black lines (average and average $\pm 2 S T D$, Fig. 9). The two curves average $\pm 2 S T D$ will be referred to as envelope curves in the following.

The same results have been obtained on each test group (table 1) but are not presented here for concision sake.

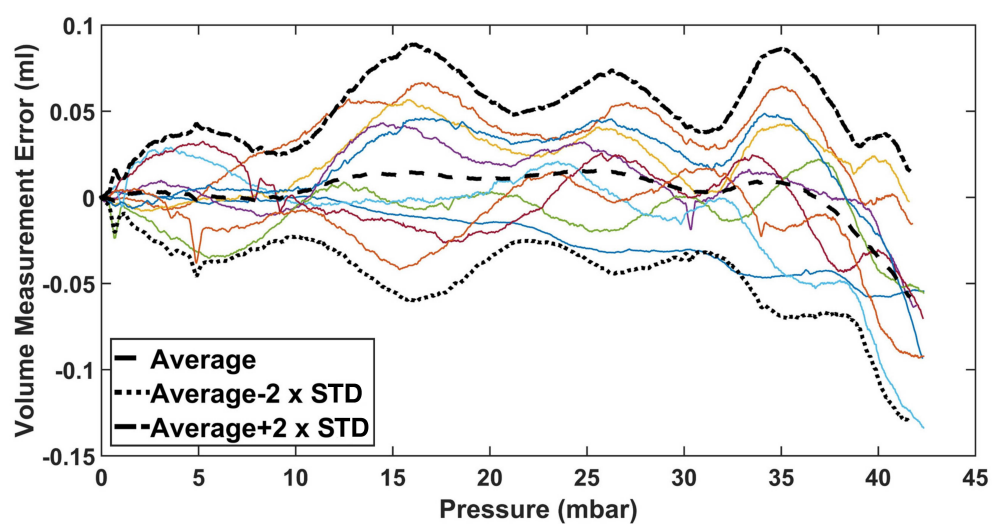

Fig. 9. Comparison of 9 diagrams of rate-based errors Error $^{R B}$, their average and STD for test group VT-1.

\section{Results and discussion}

In this section the two volume measurement errors Error ${ }^{R B}$ and Error ${ }^{\text {camera }}$ have been analyzed and compared: averages and standard deviations of the errors have been represented for all the test groups (table 1) in two different categories:

(1) Tests with the same aspiration Aperture Diameter (AD, Section 6.1).

(2) Tests with the same Syringe Diameter (SD, Section 6.2).

\subsection{Tests with the same aspiration Aperture Diameter (AD)}

Rate-based results

The rate-based volume error $\operatorname{Error}^{R B}$ for each test group with the same aperture diameter has been presented using only their envelope curves in Figs. 10a $(\mathrm{AD}=21 \mathrm{~mm})$ and $10 \mathrm{~b}(\mathrm{AD}=12.9 \mathrm{~mm})$. Theses results underline:

- The average rate-based errors Error ${ }^{R B}$ are oscillating around zero (no measurement bias) for both aspiration Aperture Diameters AD.

- The standard deviation of measurements errors can be reduced by choosing a smaller Syringe Diameter SD. 
It could also be underlined here that decreasing the Syringe Diameter SD diminishes the maximal aspirated volume $V^{\text {total }}$ out of the system (Fig. 3) and consequently reduces the maximal pressure range.

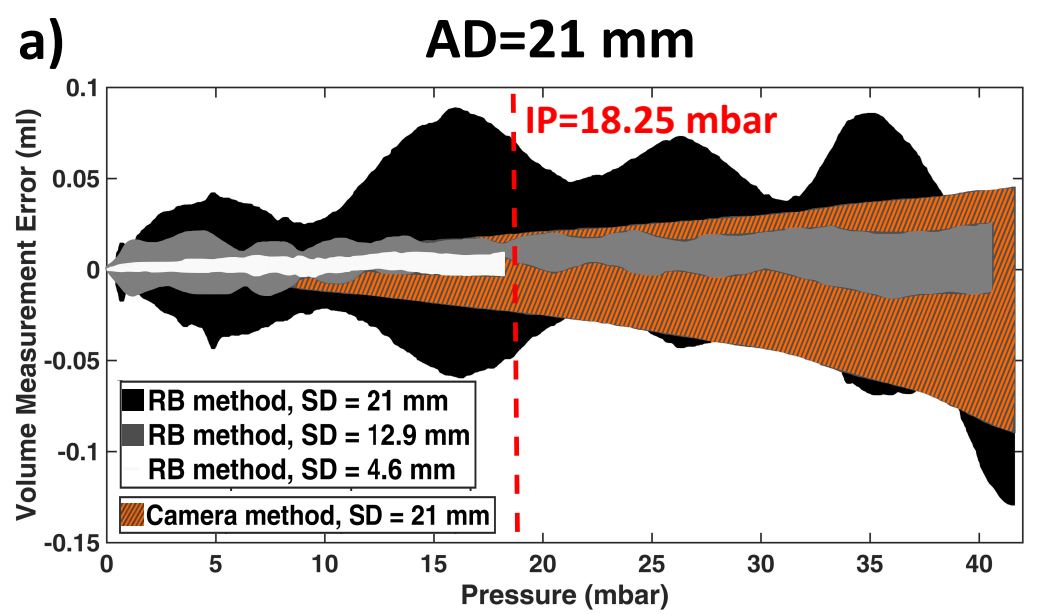

b)

$A D=12.9 \mathrm{~mm}$

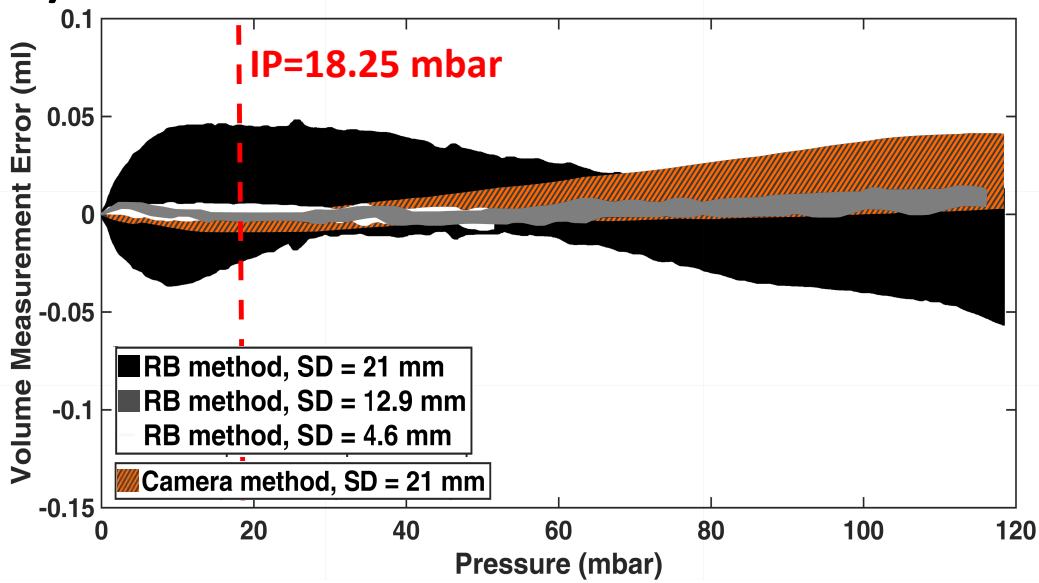

Fig. 10. Envelope curves of volume measurement errors (Error ${ }^{R B}$ and Error ${ }^{\text {camera }}$ ) versus pressure $P$ for the tests with different Syringe Diameters SD and aspiration Aperture Diameters $\mathrm{AD}=\mathrm{a}) 21 \mathrm{~mm}$ and b) $12.9 \mathrm{~mm}$.

\section{Optical results}

The envelope curves of the optical volume errors Error ${ }^{\text {camera }}$ are presented for each of the aspiration Aperture Diameter $\mathrm{AD}=21$ and $12.9 \mathrm{~mm}$ (hatched areas in 
Figs. 10a and b, respectively).

- The standard deviation of camera measurements errors increases with the pressure level and is of the same order of magnitude as the rate-based volume error Error ${ }^{R B}$.

It yet should be underlined here that by properly choosing the Syringe Diameter SD, the rate-based method can provide more accurate results than the optical method. This observation will encourage the use of the rate-based method rather than the optical method thanks to its simplicity and accuracy.

\section{Results comparison}

To compare the methods accuracy more fairly, the results have been evaluated at a the pressure (IP) of 18.25mbar (red vertical dashed line, Figs. 10a and b), which is the maximal pressure for which data is available for all the different tests. The extracted standard deviations at IP are presented as functions of the Syringe Diameter SD (Fig. 11). As previously mentioned, it can be underlined that: decreasing the Syringe Diameter SD reduces the error standard deviation. In particular, the Syringe Diameter SD can be chosen so as to obtain smaller errors than with the optical method. This conclusion is independent of the chosen aspiration aperture diameter or chosen pressure IP.

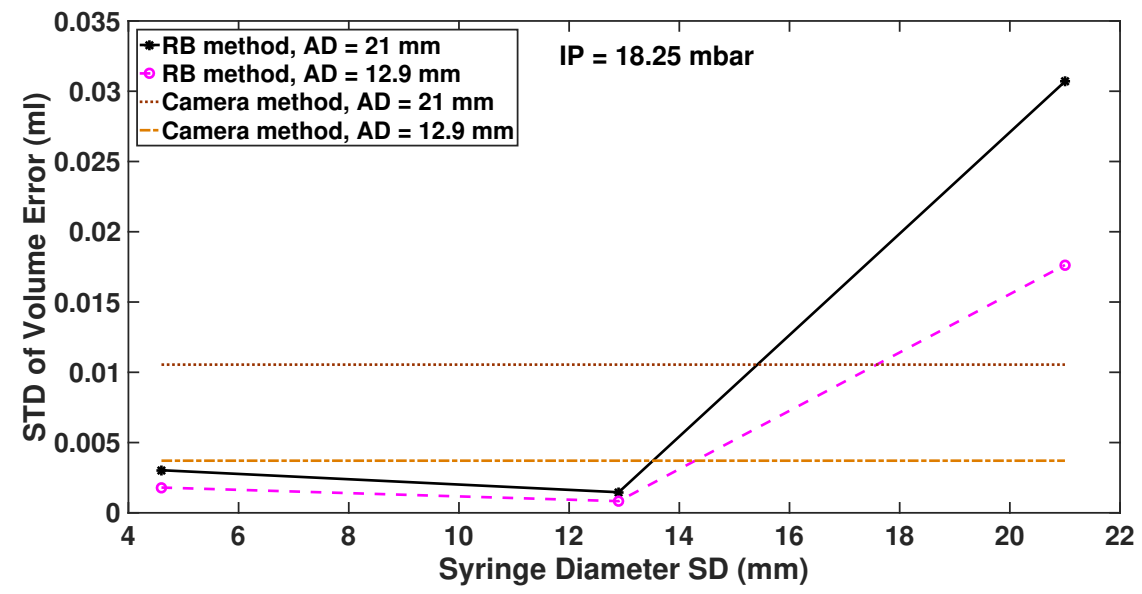

Fig. 11. STD of volume measurement errors (Error ${ }^{R B}$ and Error $\left.{ }^{\text {camera }}\right)$ versus SD for the tests with various $\mathrm{AD}$ at Iso Pressure (IP). 


\section{a)}

$\mathrm{SD}=21 \mathrm{~mm}$

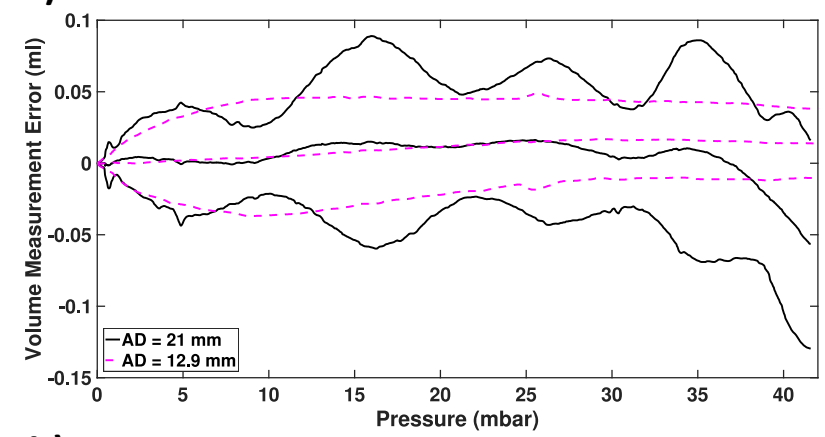

b)

$\mathrm{SD}=12.9 \mathrm{~mm}$
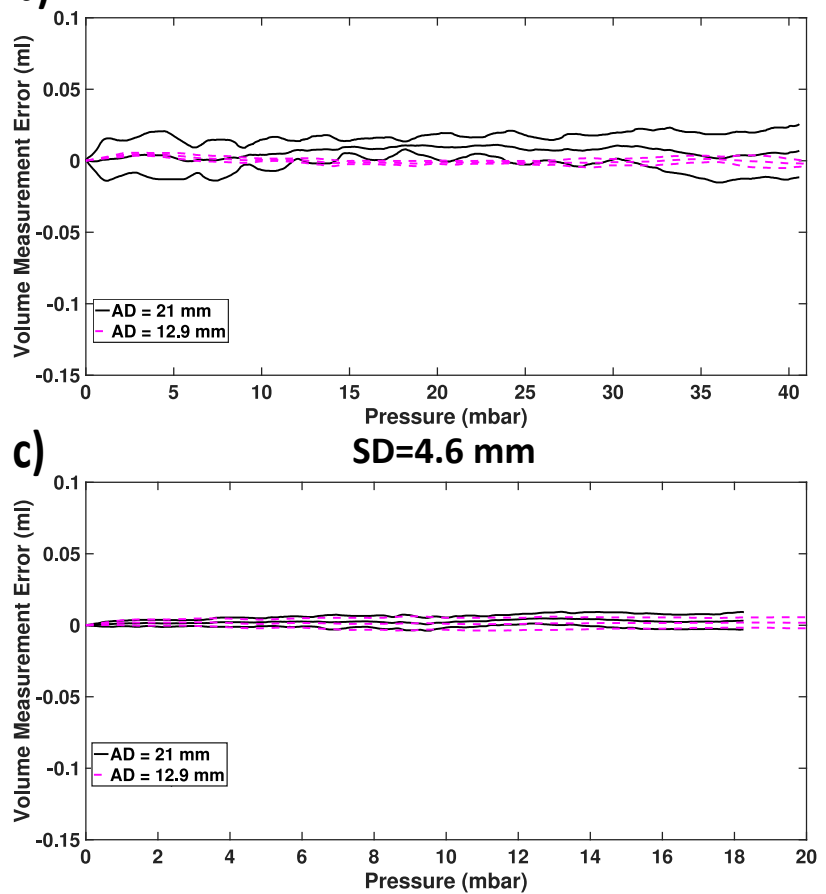

Fig. 12. Rate-based error $\operatorname{Error}^{R B}$ (average and average $\pm 2 \times \mathrm{STD}$ ) versus pressure $P$ for $\mathrm{AD}=$ 12.9 and $21 \mathrm{~mm}$ with $\mathrm{SD}=$ a) $21 \mathrm{~mm}$, b) $12.9 \mathrm{~mm}$ and c) $4.6 \mathrm{~mm}$.

\subsection{Tests with the same Syringe Diameter (SD)}

It has been underlined previously that the rate-based measurement errors mainly depend on the Syringe Diameter SD. To emphasis the impact of the aspiration Aperture Diameter AD on the errors, the results have also been studied for similar syringe diameters (Figs. 12a to c). In each graph, the observed error curves are of the same order of magnitude whatever the Aperture Diameter AD value is. The 
aspiration Aperture Diameter AD has thus almost no impact on the error of the ratebased volume measurement method $\operatorname{Error}^{R B}$. The aspiration aperture diameter can thus be chosen by taking into account only criteria as space constraints or required depth analyze.

\section{User recommendations}

To apply the rate-based volume measurement method $V_{\text {sample }}^{R B}$, a user should take into account the following considerations:

- Pay a special attention to prevent any leakage in both main and rigid tests. Before any test, applying an initial small negative pressure (in range of $1 \mathrm{mbar}$ ) and checking the pressure stability would be advisable. This initial pressure should be very small compared to the measurements pressure range. Special fixture apparatus should be design to perform the rigid test, using, for example, a caoutchouc surface clamped on the aspiration aperture.

- To avoid potential effects of the temperature on the error, the main and rigid tests should be performed in the same environment and within a short interval.

- If sterilized conditions are required during the actual test, the rigid body test should be performed after the main test to avoid any possible system contamination.

- Too high volume rate applied by the syringe pump can be a source of error due to viscoelastic behavior of either tissue or system: low enough volume rate should thus be used. Such a volume rate can be partially estimated during rigid tests performed before sterilization and by insuring that the volume rate has no effect on the obtained function $V_{\text {rigid }}^{\text {total }}(P)$. In this work, a volume rate of $0.4 \mathrm{ml} / \mathrm{min}$ has been selected. The same method can be applied while testing the material with equal or lower volume rate to ensure its behavior is rate independent. The presented methods and systems could also be used to estimate viscoelastic properties of materials, but this aspect is beyond the scope of this work.

\section{Conclusions}

An original method based on volume measurement has been developed and validated to perform aspiration tests on living soft tissues. Unlike previous aspiration devices, this method does not require the presence of camera, mirror, or any electronic part. It thus enable designing simpler, lighter, smaller and easily sterilizable systems compared to the previous proposed systems.

In particular, the proposed method requires two measurement steps: (1) measuring the system+tissue behavior and (2) measuring only the system behavior during a secondary test referred to as rigid test.

A performance assessment test-bench has been proposed to estimate the experimental bias and errors of the method. Classic optical method has also been 
implemented so as to compare each method strengths and weaknesses.

As a conclusion, the volume rate-based method proved to have similar or lower measurement error than the classic optical method provided that the syringe diameter used in the syringe pump is properly chosen and that no leakage occurs during the tests. Eventually, it has been experimentally proven that the aspiration aperture diameter has a negligible impact on the measurements error.

User recommendations have also been provided to guide any user that would be prone to use such a method.

\section{References}

1. Payan Y, Ohayon J, (eds.)Biomechanics of Living Organs: Hyperelastic constitutive laws for finite element modeling, Elsevier, Academic Press Series in Biomedical Engineering, 2017.

2. Badir S, Mazza E, Zimmermann R, Cervical softening occurs early in pregnancy: Characterization of cervical stiffness in 100 healthy women using the aspiration technique, Prenatal Diag 33:737-741, 2013.

3. Mazza E, Parra-Saavedra M, Bajka M, Gratacos E, Nicolaides K, Deprest J, In vivo assessment of the biomechanical properties of the uterine cervix in pregnancy, Prenatal Diag 34:33-41, 2014.

4. Szkely G, Surgical simulators, Min Invas Ther \& Allied Thechnol 12:14-18, 2003.

5. Gentleman E, Lay AN, Dickerson DA, Nauman EA, Livesay GA, Dee KC, Mechanical characterization of collagen fibers and scaffolds for tissue engineering, Biomaterials 24:3805-3813, 2003.

6. Snedeker JG, Bajka M, Hug JM, Niederer P, The creation of a high-fidelity finite element model of the kidney for use in trauma research, $J$ Visual Comp Animat 13:5364,2002 .

7. Hollenstein M, Jabareen M, Breitenstein S, Riener MO, Clavien PA, Bajka M, Mazza E,Intraoperative mechanical characterization of human liver, Proc. Appl. Math. Mech. 9:83-86, 2009.

8. Carter FJ, Frank TG, Davies PJ, McLean D, Cuschieri A, Measurements and modelling of the compliance of human and porcine organs, Med Image Anal 5:231-236, 2001.

9. Samur E, Sedef M, Basdogan C, Avtan L, Duzgun O, A robotic indenter for minimally invasive measurement and characterization of soft tissue response, Med Image Anal 11:361-373, 2007

10. Yao W, Yoshida K, Fernandez M, Vink J, Wapner RJ, Ananth CV, Oyen ML, Myers $\mathrm{KM}$, Measuring the compressive viscoelastic mechanical properties of human cervical tissue using indentation, $J$ Mech Behav Biomed 34:18-36, 2014.

11. Brown JD, Rosen J, Kim YS, Chang L, Sinanan MN, Hannaford B, In-vivo and in-situ compressive properties of porcine abdominal soft tissues, St Heal T 94:26-32, 2003.

12. Agache PG, Monneur C, Leveque JL, Rigal JD, Mechanical properties and Young's modulus of human skin in vivo, Arch Dermatol Res 269:221-232, 1980.

13. Diridollou S, Patat F, Gens F, Vaillant L, Black D, Lagarde JM, Gall Y, Berson M, In vivo model of the mechanical properties of the human skin under suction, Skin Res Technol 6:214-221, 2000.

14. Badir S, Mazza E, Bajka M, Objective assessment of cervical stiffness after administration of misoprostol for intrauterine contraceptive insertion, Ultrasound international open 2:63-67, 2016. 
15. Hendriks FM, Brokken D, Oomens CWJ, Bader DL, Baaijens FPT, The relative contributions of different skin layers to the mechanical behavior of human skin in vivo using suction experiments, Med Eng Phys 28:259-266, 2006.

16. Badir S, Bajka M, Mazza E, A novel procedure for the mechanical characterization of the uterine cervix during pregnancy, J Mech Behav Biomed 27:143-153, 2013.

17. Nava A, Mazza E, Furrer M, Villiger P, Reinhart WH, In vivo mechanical characterization of human liver, Med Image Anal 12:203-206, 2008.

18. Luboz V, Promayon E, Payan Y, Linear elastic properties of the facial soft tissues using an aspiration device: towards patient specific characterization, Ann Biomed Eng 42:2369-2378, 2014.

19. Kauer M, Vuskovic V, Dual J, Szekely G, Bajka M, Inverse finite element characterization of soft tissues, Med Image Anal 6:275-287, 2002.

20. Schiavone P, Boudou T, Promayon E, Perrier P, Payan Y, A light sterilizable pipette device for the in vivo estimation of human soft tissues constitutive laws, Proc. 30th Annual Int. IEEE EMBS Conf., Canada, British Columbia, Vancouver, pp. 4298-4301, 2008.

21. Schiavone P, Chassat F, Boudou T, Promayon E, Valdivia F, Payan Y, In vivo measurement of human brain elasticity using a light aspiration device, Med Image Anal 13:673-678, 2009.

22. Schiavone P, Promayon E, Payan Y, LASTIC: a Light Apiration device for in vivo Soft TIssue Characterization, Proc. 5th Int. Symp. Biomedical Simulation ISBMS, Series: lecture notes in computer science, USA, pp. 1-10, 2010.

23. Luboz V, Promayon E, Chagnon G, Alonso T, Favier D, Barthod C, Payan Y, Validation of a light aspiration device for in vivo soft tissue characterization (LASTIC), in Payan Y (ed.), Soft tissue biomechanical modeling for computer assisted surgery, Springer, pp. 243-256, 2012.

24. Hollenstein M, Bugnard G, Joos R, Kropf S, Villiger P, Mazza E, Towards laparoscopic tissue aspiration, Med Image Anal 17:1037-1045, 2013.

25. Weickenmeier J, Jabareen M, Mazza E, Suction based mechanical characterization of superficial facial soft tissues, J Biomech 48:4279-4286, 2015.

26. Florentin E, Lubineau G, Identification of the parameters of an elastic material model using the constitutive equation gap method, Comput Mech 46:521-531, 2010. 